\title{
THE AVERAGE RESOLVING NUMBER OF GRAPHS
}

\author{
Saravanan $\mathrm{P}^{1^{*}}$, Sujatha $\mathrm{R}^{2}$ and Sundareswaran $\mathrm{R}^{3}$ \\ ${ }^{1}$ Department of Mathematics, G.B Pant Government Engineering College, \\ New Delhi, India \\ ${ }^{2,3}$ Department of Mathematics, SSN Engineering College, Chennai, India \\ ${ }^{1}$ saran202001@gmail.com, ${ }^{2}$ sujathar@ssn.edu.in, ${ }^{3}$ sundareswaranr@ ssn.edu.in
}

Abstract - Let $G$ be a finite, simple, undirected and connected graph with vertex set $V(G)$. For an ordered subset $W=\left\{w_{1}, w_{2}, \ldots w_{k}\right\}$ of vertices and a vertex ' $v$ ' in $G$, the representation of ' $v$ ' with respect to $W$ is the ordered $k$-tuple $r(v \mid W)=$ $\left(d\left(v, w_{1}\right), d\left(v, w_{2}\right), \cdots, d\left(v, w_{k}\right)\right)$, where $d(x, y)$ is the distance between the vertices ' $x$ ' and ' $y$ '. The set $W$ is a resolving set (or locating set) for $G$ if every two vertices of $G$ have distinct representations . The metric dimension of $G(\operatorname{dim}(G))$ is the minimum cardinality of a resolving set for $G$. The resolving set containing minimum number of vertices is called a basis (or reference set) for $G$. In this paper, we introduce a new resolving parameter, average resolving number $\left(r_{a v}(G)\right)$ of $G$ and a new graph, namely resolving excellent graph, both depends on minimum cardinality of the resolving set of the graph $G$ containing each element of $V(G)$. The average resolving number of some standard graphs, product graphs, m-bismuth chain and m-lead chloride chain are found and we investigate the resolving excellent graphs. We find the upper Bounds of average resolving number in terms of order(n) and diameter(d) of the connected graph G. The need for this new resolving parameter $\left(r_{a v}(G)\right)$ is explained using the isomers of alkanes, which are chemical compounds having same molecular formula but different chemical structures.

Keywords - Metric dimension, Resolving Set, Resolving Number, Average Resolving Number, Resolving Excellent Graph, Bismuth Tri-Iodide, Lead Chloride

\section{INTRODUCTION}

Let $G$ be a finite, simple, undirected and connected graph with vertex set $V(G)$. The distance $d(u, v)$ between two vertices of ' $u$ ' and ' $v$ ' of $G$ is the length of the shortest $u-v$ path in $G$. For an ordered subset $W=\left\{w_{1}, w_{2}, \ldots, w_{k}\right\}$ of vertices and a vertex $v$ in $G$, the representation of $v$ with respect to $W$ is the ordered $k$-tuple $r(v \mid \mathrm{W})=$ $\left(d\left(v, w_{1}\right), d\left(v, w_{2}\right), \cdots, d\left(v, w_{k}\right)\right)$, where $d(x, y)$ is the distance between the vertices ' $x$ ' and ' $y$ '. The set $W$ is called a resolving set of $G$ if every two vertices of $G$ have distinct representations. For a given ordered set of vertices $W=\left\{w_{1}, w_{2}, \ldots, w_{k}\right\}$ of a graph $\mathrm{G}$, the $i$ th component of $r(v \mid W)$ is 0 iff $v=w_{i}$. Thus, to prove that $W$ is a resolving set, it is suffice to show $r(x \mid W) \neq r(y \mid W)$ for any two distinct vertices $x, y \in V(G) \backslash W$. The metric dimension of $G, \operatorname{dim}(G)$ is the minimum cardinality of a resolving set for $G$. A resolving set containing minimum number of vertices is called a basis (or reference set) for $G$. The resolving number $\operatorname{res}(G)$ of a connected graph $G$ is the minimum $k$ such that every $k$-set $W$ of vertices of $G$ is also a resolving set of $G$.

Resolving set (or locating set) and metric dimension were first introduced by Slater[1]

Received: May 17, 2019

Reviewed: August 28, 2019

Accepted: August 30, 2019

* Corresponding Author

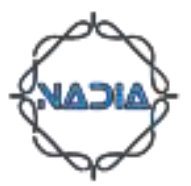


in 1945, and independently by Harary and Melter[2] in 1976. Much later, Chartrand et al. in the year 2000, defined the concept of resolving number [3].

Various applications of resolving sets and metric dimensions of graphs have been studied, including the structure of chemical compound [4], drug discovery [5], coin weighing problem [6], network discovery and verification [7], robot navigation [8] and and strategies for mastermind game [9]. There exists by now an extensive literature on resolving sets and resolving parameters related to them,for instance, upper dimension, partition dimension, resolving number, total resolving number, weak total resolving number, nearly resolving number, strong total resolving number, non-isolated resolving number, star resolving number, path resolving number to name a few.

Resolving parameters associated to a finite dimensional vector space are also studied by various authors. For instance, recently, the resolving parameters such as metric dimension and partition dimension for the non-zero component graph, associated to a finite vector space, are discussed in [10].

Chartrand et al. [3] have defined a resolving parameter, namely upper dimension and the concept of minimal resolving sets. A resolving set $W$ of $G$ is said to be the minimal if no proper subset of $W$ is a resolving set.The maximum cardinality of a minimal resolving set is the upper dimension $\operatorname{dim}^{+}(G)$ and they have proved that $1 \leq$ $\operatorname{dim}(G) \leq \operatorname{dim}^{+}(G) \leq \operatorname{res}(G) \leq n-1$ for every non-trivial connected graph $G$ of order n. The upper dimension $\operatorname{dim}^{+}(G)$ gives an insight of how large the set of resolving sets of a graph is. They have also shown that $\operatorname{dim}^{+}(G)=\operatorname{res}(G)=n-1$ if and only if $G=K_{n}$. and determined the upper dimensions of some well known graphs.

The two parameters, $\operatorname{dim}(G)$ and $\operatorname{dim}^{+}(G)$ are minimum cardinality of a resolving set and maximum cardinality of a minimal resolving set respectively. An element $v \in$ $V(G)$ may or may not belongs to these resolving sets. We have defined a new resolving parameter, namely average resolving number $r_{a v}(G)$ as the average of all the minimum cardinalities of the resolving set of $G$ containing each $u \in V(G)$. That is, the minimum cardinality of the resolving set containing each element in $V(G)$ has been taken into consideration.

\section{AVERAGE RESOLVING NUMBER AND RESOLVING EXCELLENT GRAPHS}

DEFINITION 2.1.

The average resolving number of a graph $G$, with vertex set $V(G)$, denoted by $r_{a v}(G)$ is defined as

$$
r_{a v}(G)=\frac{1}{|V(G)|} \sum_{u \in V(G)} r_{u}(G)
$$

where $r_{u}(G)$ is the minimum cardinality of a resolving set of $G$ containing $u$.

From the above definition, it is easy to verify that $r_{a v}(G)$ lies between $\operatorname{dim}(G)$ and $\operatorname{res}(G)$.

\section{DEFINITION 2.2 .}

A graph $G$ is said to be resolving excellent if $r_{u}(G)=\operatorname{dim}(G)$ for all $u \in V(G)$.

EXAMPLE 2.2.1 : To illustrate these concepts, consider the graph $G$ of Figure 1 and let $W_{1}=\{v, w\}$. The representations for the vertices of $V(G) \backslash W_{1}$ with respect to $W_{1}$ are: $r\left(x \mid W_{1}\right)=(1,1), r\left(y \mid W_{1}\right)=(2,1), r\left(z \mid W_{1}\right)=(2,2)$. Since these representations are distinct, $W_{1}$ is a resolving set for $G$. Since no singleton set is a resolving set of G, 
$W_{1}$ is a minimum resolving set of $\mathrm{G}$ and hence $\operatorname{dim}(G)=2, r_{v}(G)=r_{w}(G)=2$.

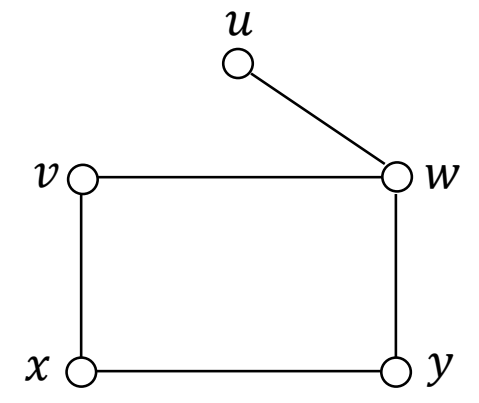

Fig. $1 \quad$ A graph $G$ with $\operatorname{dim}(G)=2$ and $r_{a v}(G)=2.2$

By a case-by-case analysis, we can find that, $W_{2}=\{v, z\}, W_{3}=\{y, z\}$ and $W_{4}=$ $\{y, z, x\}$ are minimum resolving sets containing $z, y$ and $x$ respectively of $G$.Thus, $r_{z}(G)=r_{y}(G)=2$ and $r_{x}(G)=3$.The representations for the vertices of $V(G) \backslash W_{i}$ with respect to $W_{i}(i=2,3,4)$ are given in Table I.

Hence, $r_{a v}(G)=\frac{(2+2+2+2+3)}{5}=\frac{11}{5}$ and $G$ is not resolving excellent as $r_{w}(G) \neq$ $\operatorname{dim}(G)$.

Table I. The Representations $r\left(u \mid W_{i}\right)$ for $u \in V(G) \backslash W_{i}$ with respect to $W_{i}$ ( $i=$ $2,3,4)$

\begin{tabular}{|c|c|c|c|c|c|}
\hline $\boldsymbol{u}$ & $\boldsymbol{r}\left(\boldsymbol{u} \mid \boldsymbol{W}_{\mathbf{2}}\right)$ & $\boldsymbol{u}$ & $\boldsymbol{r}\left(\boldsymbol{u} \mid \boldsymbol{W}_{\mathbf{3}}\right)$ & $\boldsymbol{u}$ & $\boldsymbol{r}\left(\boldsymbol{u} \mid \boldsymbol{W}_{\mathbf{4}}\right)$ \\
\hline$w$ & $(2,2)$ & $v$ & $(2,2)$ & $v$ & $(2,2,1)$ \\
\hline$x$ & $(1,1)$ & $w$ & $(1,2)$ & $w$ & $(1,2,1)$ \\
\hline$y$ & $(2,1)$ & $x$ & $(1,1)$ & & \\
\hline
\end{tabular}

EXAMPLE 2.2.2: In this example, we show that there exists graphs with equal number of vertices and edges whose metric dimensions are same but the average resolving numbers are different. Consider the graphs $G_{1}$ and $G_{2}$ in Fig 2. Both $G_{1}$ and $G_{2}$ has 5 vertices and 6 edges.
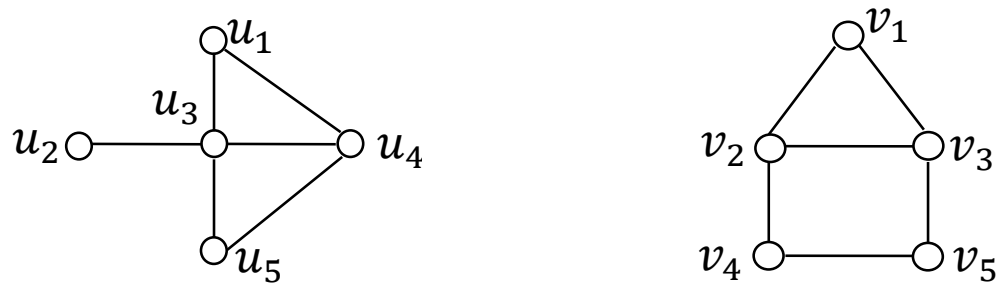

Fig. 2 Graphs $G_{1}$ and $G_{2}$

For $G_{1}, W_{1}=\left\{u_{1}, u_{2}\right\}, W_{2}=\left\{u_{1}, u_{2}, u_{3}\right\}, W_{3}=\left\{u_{1}, u_{4}\right\}$ and $W_{4}=\left\{u_{2}, u_{5}\right\}$ are minimum resolving sets containing $u_{1}\left(u_{2}\right), u_{3}, u_{4}$ and $u_{5}$ respectively and hence, $r_{u_{1}}\left(G_{1}\right)=2 ; r_{u_{2}}\left(G_{1}\right)=2 ; r_{u_{3}}\left(G_{1}\right)=3 ; r_{u_{4}}\left(G_{1}\right)=2 ; r_{u_{5}}\left(G_{1}\right)=2$. Hence $\operatorname{dim}\left(G_{1}\right)=$ 2 and $r_{a v}\left(G_{1}\right)=2.2$. The representations for the vertices in $V\left(G_{1}\right) \backslash W_{i}$ with respect to $W_{i}(i=1,2,3,4)$ are given in Table II. 
Table II. The Representations $r\left(u \mid W_{i}\right)$ for $u \in V\left(G_{1}\right) \backslash W_{i}$ with respect to $W_{i}$ (i= $1,2,3,4)$

\begin{tabular}{|c|c|c|c|c|c|c|c|}
\hline $\boldsymbol{u}$ & $\boldsymbol{r}\left(\boldsymbol{u} \mid \boldsymbol{W}_{\mathbf{1}}\right)$ & $\boldsymbol{u}$ & $\boldsymbol{r}\left(\boldsymbol{u} \mid \boldsymbol{W}_{\mathbf{2}}\right)$ & $\boldsymbol{u}$ & $\boldsymbol{r}\left(\boldsymbol{u} \mid \boldsymbol{W}_{\mathbf{3}}\right)$ & $\boldsymbol{u}$ & $\boldsymbol{r}\left(\boldsymbol{u} \mid \boldsymbol{W}_{\mathbf{4}}\right)$ \\
\hline$u_{3}$ & $(1,1)$ & $u_{4}$ & $(1,2,1)$ & $u_{2}$ & $(2,2)$ & $u_{1}$ & $(2,2)$ \\
\hline$u_{4}$ & $(1,2)$ & $u_{5}$ & $(2,2,1)$ & $u_{3}$ & $(1,1)$ & $u_{3}$ & $(1,1)$ \\
\hline$u_{5}$ & $(2,2)$ & & & $u_{5}$ & $(2,1)$ & $u_{4}$ & $(2,1)$ \\
\hline
\end{tabular}

For $G_{2}, W_{1}=\left\{v_{1}, v_{2}\right\}, W_{2}=\left\{v_{1}, v_{3}\right\}, W_{3}=\left\{v_{1}, v_{4}\right\}$ and $W_{4}=\left\{v_{1}, v_{5}\right\}$ are minimum resolving sets containing $v_{1}\left(v_{2}\right), v_{3}, v_{4}$ and $v_{5}$ respectively and hence $r_{v_{1}}\left(G_{2}\right)=2 ; r_{v_{2}}\left(G_{2}\right)=2 ; r_{v_{3}}\left(G_{2}\right)=2, r_{v_{4}}\left(G_{2}\right)=2$ and $r_{v_{5}}\left(G_{2}\right)=2$. Hence $\operatorname{dim}\left(G_{2}\right)=2$ and $r_{a v}\left(G_{2}\right)=2$. The representations for the vertices of $V\left(G_{2}\right) \backslash W_{i}$ with respect to $W_{i}(i=1,2,3,4)$ are given in Table III.

Table III. The Representations $r\left(v \mid W_{i}\right)$ for $v \in V\left(G_{2}\right) \backslash W_{i}$ with respect to $W_{i}$ (i= $1,2,3,4)$

\begin{tabular}{|c|c|c|c|c|c|c|c|}
\hline $\boldsymbol{v}$ & $\boldsymbol{r}\left(\boldsymbol{v} \mid \boldsymbol{W}_{\mathbf{1}}\right)$ & $\boldsymbol{v}$ & $\boldsymbol{r}\left(\boldsymbol{v} \mid \boldsymbol{W}_{\mathbf{2}}\right)$ & $\boldsymbol{v}$ & $\boldsymbol{r}\left(\boldsymbol{v} \mid \boldsymbol{W}_{\mathbf{3}}\right)$ & $\boldsymbol{v}$ & $\boldsymbol{r}\left(\boldsymbol{v} \mid \boldsymbol{W}_{\mathbf{4}}\right)$ \\
\hline$v_{3}$ & $(1,1)$ & $v_{2}$ & $(1,1)$ & $v_{2}$ & $(1,1)$ & $v_{2}$ & $(1,2)$ \\
\hline$v_{4}$ & $(2,1)$ & $v_{4}$ & $(2,2)$ & $v_{3}$ & $(1,2)$ & $v_{3}$ & $(1,1)$ \\
\hline$v_{5}$ & $(2,2)$ & $v_{5}$ & $(2,1)$ & $v_{5}$ & $(2,1)$ & $v_{4}$ & $(2,1)$ \\
\hline
\end{tabular}

Hence, both $G_{1}$ and $G_{2}$ have the same metric dimension of 2 , but have different average resolving number.

Since, $r_{u_{3}}\left(G_{1}\right) \neq \operatorname{dim}\left(G_{1}\right)$ for $u_{3} \in V\left(G_{1}\right), G_{1}$ is not resolving excellent. But, as $r_{v}\left(G_{2}\right)=\operatorname{dim}\left(G_{2}\right)$ for all $v \in V\left(G_{2}\right), G_{2}$ is resolving excellent.

In the next example we give two chemical graphs such that whose $\operatorname{dim}(G)$ and $\operatorname{dim}^{+}(G)$ are same but their average resolving numbers are different. For this, we use the following definitions, introduced in [4]. A vertex of degree atleast three in a graph $G$ will be called a major vertex of $G$. Any end-vertex $u$ of $G$ is said to be a terminal vertex of a major vertex $v$ of $G$ if $d(u, v)<d(u, w)$ for every other major vertex $w$ of $G$. The terminal degree, denoted by $\operatorname{ter}(v)$ of a major vertex $v$ is the number of terminal vertices of $v$. A major vertex $v$ of $G$ is an exterior major vertex of $G$ if it has positive terminal degrees. Let $\sigma(G)$ denote the sum of the terminal degrees of the major vertices of $G$, and let $\operatorname{ex}(G)$ denote the number of exterior major vertex of $G$.

EXAMPLE 2.2.3: A fundamental problem in chemical graph theory is to give mathematical representations for a set of chemical compounds in such a way that distinct compounds have distinct representations [11]. Compounds that have same molecular formula but have different chemical structures are called isomers and alkanes are molecules consisting of only carbon and hydrogen atoms. The isomers of alkanes can have different physical and chemical properties. Since the isomers of alkanes are structurally different, we need a resolving parameter which gives different values for these isomers. The general formula of alkane is $C_{n} H_{2 n+2}, n$ is a positive integer and the chemical graphs of isomers of alkanes are trees.For $\mathrm{n}=4$, the alkane is $C_{4} H_{10}$ and the number of possible isomers is 2,called butane and isobutane as shown in Fig.3(a) and Fig.3(b) respectively.

The list of all major vertices, terminal vertices of major vertices, terminal degree and exterior major vertices of $T_{1}$ and $T_{2}$ are given in Table IV and Table $\mathrm{V}$ respectively. 
<smiles>CCCC</smiles>

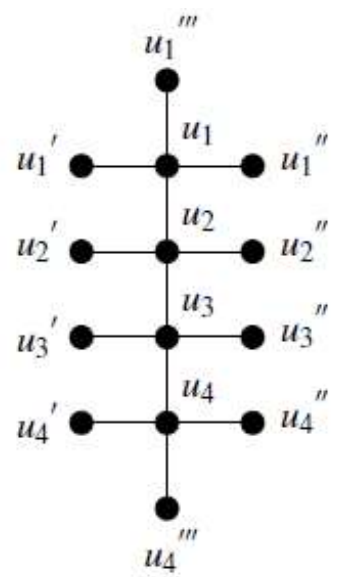

Fig. 3(a). Butane and its Chemical graph $T_{1}$<smiles>CC(C)C</smiles>

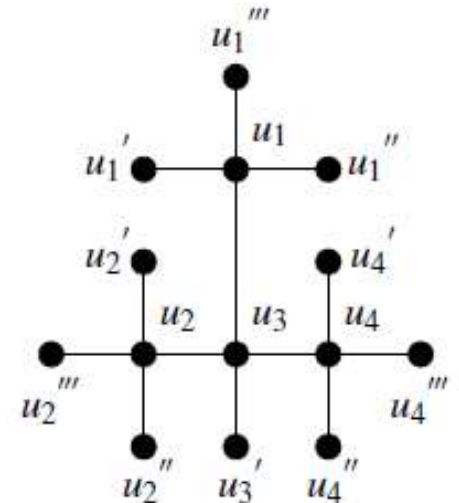

Fig. 3(b). Isobutane and its Chemical graph $T_{2}$

Table IV. The List Of All Major Vertices, Terminal Vertices Of Major Vertices, Terminal Degree and Exterior Major Vertices Of $T_{1}$

\begin{tabular}{|c|c|c|c|}
\hline $\begin{array}{c}\text { Major Vertices of } \\
\boldsymbol{T}_{\mathbf{1}}\end{array}$ & $\begin{array}{c}\text { Terminal Vertices of } \\
\boldsymbol{u}_{\boldsymbol{i}}(\boldsymbol{i}=\mathbf{1}, \mathbf{2}, \mathbf{3}, \mathbf{4})\end{array}$ & $\begin{array}{c}\operatorname{ter}\left(\boldsymbol{u}_{\boldsymbol{i}}\right) \\
(\boldsymbol{i}=\mathbf{1}, \mathbf{2}, \mathbf{3}, \mathbf{4})\end{array}$ & $\begin{array}{c}\text { Exterior Major } \\
\text { Vertices of } \boldsymbol{T}_{\mathbf{1}}\end{array}$ \\
\hline$u_{1}$ & $u_{1}^{\prime}, u_{1}^{\prime \prime}, u_{1}^{\prime \prime \prime}$ & 3 & $u_{1}$ \\
\hline$u_{2}$ & $u_{2}^{\prime}, u_{2}{ }^{\prime \prime}$ & 2 & $u_{2}$ \\
\hline$u_{3}$ & $u_{3}{ }^{\prime \prime}, u_{3}{ }^{\prime \prime}$ & 2 & $u_{3}$ \\
\hline$u_{4}$ & $u_{4}^{\prime}, u_{4}^{\prime \prime}, u_{4}{ }^{\prime \prime \prime}$ & 3 & $u_{4}$ \\
\hline
\end{tabular}

Chartrand et al. [3] have proved that if $T$ is a tree that is not a path, then $\operatorname{dim}(T)=$ $\operatorname{dim}^{+}(T)=\sigma(T)-\operatorname{ex}(T)$. Here, since both $T_{1}$ and $T_{2}$ are trees and are not paths, from Table IV, $\sigma\left(T_{1}\right)=10$ and $\operatorname{ex}\left(T_{1}\right)=4$ and hence $\operatorname{dim}\left(T_{1}\right)=\operatorname{dim}^{+}\left(T_{1}\right)=6$. From Table $\mathrm{V}, \sigma\left(T_{2}\right)=10$ and $\operatorname{ex}\left(T_{2}\right)=4$ and hence $\operatorname{dim}\left(T_{2}\right)=\operatorname{dim}^{+}\left(T_{2}\right)=6$. Thus, both $T_{1}$ and $T_{2}$ have same dimension and upper dimension.

Chartrand et al.[4] have shown that, if $T$ is a tree that is not a path, then $\operatorname{dim}(T)=$ $\sigma(T)-e x(T)$ by constructing a resolving set $W$ of vertices of $T$ by placing every terminal vertex, except one, of each exterior major vertex of $T$ in $W$ and every other resolving set should contain all the elements of $W$. Using this result, we can construct a resolving set $W$ of $T_{1}$ by placing any two terminal vertices of $u_{1}$, any one terminal vertex of $u_{2}$, any one terminal vertex of $u_{3}$ and any two terminal vertices of $u_{4}$. Thus, this set will consists of 6 vertices and hence $r_{u}\left(T_{1}\right)=6$ for every terminal vertex $u \in$ 
$V\left(T_{1}\right)$. Note that every other resolving set should contain all the elements of $W$ and hence $r_{u}\left(T_{1}\right)=7$ for every major vertex $u \in V\left(T_{1}\right)$. Hence $r_{a v}\left(T_{1}\right)=\frac{(10 \times 6)+(4 \times 7)}{14}=$ $\frac{88}{14}$. Similarly for $T_{2}$, we can prove that $r_{a v}\left(T_{2}\right)=\frac{(9 \times 6)+(5 \times 7)}{14}=\frac{89}{14}$.

Table V. The List Of All Major Vertices, Terminal Vertices of Major Vertices, Terminal Degree and Exterior Major Vertices of $T_{2}$

\begin{tabular}{|c|c|c|c|}
\hline $\begin{array}{c}\text { Major Vertices of } \\
\boldsymbol{T}_{\mathbf{2}}\end{array}$ & $\begin{array}{c}\text { Terminal Vertices of } \\
\boldsymbol{v}_{\boldsymbol{i}}(\boldsymbol{i}=\mathbf{1}, \mathbf{2}, \mathbf{3}, \mathbf{4})\end{array}$ & $\begin{array}{c}\operatorname{ter}\left(\boldsymbol{v}_{\boldsymbol{i}}\right) \\
(\boldsymbol{i}=\mathbf{1}, \mathbf{2}, \mathbf{3}, \mathbf{4})\end{array}$ & $\begin{array}{c}\text { Exterior Major } \\
\text { Vertices of } \boldsymbol{T}_{\mathbf{2}}\end{array}$ \\
\hline$v_{1}$ & $v_{1}^{\prime}, v_{1}^{\prime \prime}, v_{1}^{\prime \prime \prime}$ & 3 & $v_{1}$ \\
\hline$v_{2}$ & $v_{2}^{\prime}, v_{2}^{\prime \prime}, v_{2}^{\prime \prime \prime}$ & 3 & $v_{2}$ \\
\hline$v_{3}$ & $v_{3}{ }^{\prime \prime}$ & 1 & $v_{3}$ \\
\hline$v_{4}$ & $v_{4}^{\prime}, v_{4}^{\prime \prime}, v_{4}^{\prime \prime \prime}$ & 3 & $v_{4}$ \\
\hline
\end{tabular}

Since, $r_{u_{3}}\left(T_{1}\right) \neq \operatorname{dim}\left(T_{1}\right)$ for $u_{3} \in V\left(T_{1}\right)$ and $r_{v}\left(T_{2}\right)=\operatorname{dim}\left(T_{2}\right)$ for all $v \in$ $V\left(T_{2}\right), T_{1}$ is not resolving excellent whereas $T_{2}$ is resolving excellent.

In general, the metric dimension, and the upper dimension of these isomers are not all different and hence, average resolving number helps to analyze the structural properties of these isomers.

Now, we give the upper bounds for average resolving number in terms of order $(n)$ and diameter $(d)$ of the connected graph $G$.

THEOREM 2.1. If $G$ is a connected graph of order $n \geq 2$ and diameter $d$, then $\operatorname{dim}(G) \leq r_{a v}(G) \leq(n-d)+\frac{d(d-1)}{n}$.

Proof. First, we establish the lower bound. Since $\operatorname{dim}(G) \leq r_{u}(G)$, for all $u \in V(G)$, we have $\operatorname{dim}(G) \leq r_{a v}(G)$.

Next, let us prove the upper bound. Consider the vertices $u$ and $v$ of $G$ such that $d(u, v)=d$, the diameter of $G$. Let $u=u_{0}, u_{1}, \cdots, u_{d}=v$ be a $u-v$ path of length $d$ in $G$ and let $W=V(G)-\left\{u_{1}, u_{2}, \cdots, u_{d}\right\}$. Note that $u=u_{0} \in W$ and $d\left(u, u_{i}\right)=i$, for $1 \leq i \leq d$. Therefore, $W$ is a resolving set of cardinality $n-d$ for $G$ and so $r_{x}(G) \leq(n-d)$, for all $x \in W$. It is easy to verify that $r_{y}(G) \leq(n-1)$, for all $d$ elements $y \in(V(G)-W(G))$. Hence,

$$
\begin{aligned}
r_{a v}(G) & =\frac{1}{n}\left\{\sum_{x \in W} r_{x}(G)+\sum_{y \in(V-W)} r_{y}(G)\right\} \\
& \leq \frac{1}{n}\left\{\sum_{x \in W}(n-d)+\sum_{y \in(V-W)}(n-1)\right\} \\
& =\frac{1}{n}\{(n-d)(n-d)+(n-1) d\} \\
& =(n-d)+\frac{d(d-1)}{n}
\end{aligned}
$$

REMARK : The inequality given in the above Theorem can be strict. For example, the graph $\mathrm{G}$ of Fig.1 has order $n=5$, diameter $d=3, \operatorname{dim}(G)=2, r_{a v}(G)=2.4$ and $(n-d)+\frac{d(d-1)}{n}=3.2$. Thus, $\operatorname{dim}(G)<r_{a v}(G)<(n-d)+\frac{d(d-1)}{n}$.

THEOREM 2.2. A graph $G$ is resolving excellent if and only if $r_{a v}(G)=\operatorname{dim}(G)$.

Proof. Suppose $G$ is a graph of order $n$ and $G$ is resolving excellent. i.e., $r_{u}(G)=$ $\operatorname{dim}(G)$, for all $u \in G$. By the definition of average resolving number, $r_{a v}(G)=$ 
$\frac{1}{n} \sum_{u \in V(G)} r_{u}(G)=\frac{1}{n} \sum_{u \in V(G)} \operatorname{dim}(G)=\operatorname{dim}(G)$. Conversely,assume that $r_{a v}(G)=$ $\operatorname{dim}(G)$. By the definition of average resolving number, $n \times r_{a v}=\sum_{u \in V(G)} r_{u}(G)=n \times$ $\operatorname{dim}(G)$ and hence $r_{u}(G)=\operatorname{dim}(G)$, for all $u \in G$.

The next theorem gives some special graphs which are resolving excellent.

\section{THEOREM 2.3.}

1. For the path $P_{n}, n \geq 2, \operatorname{dim}\left(P_{n}\right)=1, r_{a v}\left(P_{n}\right)=2-\frac{2}{n}$ and $P_{n}$ is resolving excellent if and only if $n=1$

2. For the cycle $C_{n}, n \geq 3, \operatorname{dim}\left(C_{n}\right)=r_{a v}\left(C_{n}\right)=2$ and hence $C_{n}$ is resolving excellent for all $n \geq 3$.

3. For the complete bipartite graph $K_{m, n}, m, n \geq 2, \operatorname{dim}\left(K_{m, n}\right)=r_{a v}\left(K_{m, n}\right)=m+$ $n-2$ and hence $K_{m, n}, m, n \geq 2$ is resolving excellent.

4. For the complete graph $K_{n}, n \geq 2, \operatorname{dim}\left(K_{n}\right)=r_{a v}(G)=(n-1)$ and hence $K_{n}$ is resolving excellent.

Now we give some theorem without proof that gives some special graphs which are not resolving excellent.

\section{THEOREM 2.4.}

1. For the graph $K_{1, n-1}, n \geq 4, \operatorname{dim}\left(K_{1, n-1}\right)=n-2, \quad r_{a v}\left(K_{1, n-1}\right)=\frac{(n-1)^{2}}{n}$ and $K_{1, n-1}$ is not resolving excellent.

2. For the double star graph $S_{m, n}, \operatorname{dim}\left(S_{m, n}\right)=m+n-4, r_{a v}\left(S_{m, n}\right)=(m+n-$ $4)+\frac{2}{(m+n)}$ and hence $S_{m, n}$ is not resolving excellent.

3. For the Friendship graph $F_{n}$ with $n$-triangles, $n \geq 2, \operatorname{dim}\left(F_{n}\right)=n, r_{a v}\left(F_{n}\right)=n+$ $\frac{1}{(2 n+1)}$ and hence $F_{n}$ is not resolving excellent.

4. For the broom graph $B_{n, d}, \operatorname{dim}\left(B_{n, d}\right)=n-d, r_{a v}\left(B_{n, d}\right)=(n-d)+\frac{1}{n}$ and hence $B_{n, d}$ is not resolving excellent.

5. Let $T$ be a tree order $n$, that is not a path. Then, $r_{a v}(T)=\operatorname{dim}(T)+\frac{\operatorname{ex}(T)}{n}$ and hence $T$ is not resolving excellent.

COROLLARY 2.4.1 For every positive integer $k$, there exists a resolving excellent graph $G$ with average resolving number $k$. For: let $G=K_{k+1}, r_{a v}(G)=k$.

\section{RESOLVING EXCELLENCY OF PRODUCT GRAPHS}

In this section, we find the average resolving number of some product graphs and prove that if $H$ is a connected graph and $K_{2}$ is a path of order 2, then $r_{a v}(H) \leq$ $r_{a v}\left(H \times K_{2}\right) \leq r_{a v}(H)+1$.

THEOREM 3.1. If $P_{n}$ is a path graph of length $n(n \geq 2)$ and $K_{2}$ is a path order 2, then $\left(P_{n} \times K_{2}\right)$ is not resolving excellent.

Proof. Let $G=P_{n} \times K_{2} \quad$ and $\quad V(G)=\left\{\left(u_{1}, v_{1}\right),\left(u_{2}, v_{1}\right), \cdots,\left(u_{n}, v_{1}\right),\left(u_{1}, v_{2}\right)\right.$, $\left.\left(u_{2}, v_{2}\right), \cdots,\left(u_{n}, v_{2}\right)\right\}$. It is easy to verify that $W_{1}=\left\{\left(u_{1}, v_{1}\right),\left(u_{1}, v_{2}\right)\right\}$ and $W_{2}=$ $\left\{\left(u_{n}, v_{1}\right),\left(u_{n}, v_{2}\right)\right\}$ are minimum resolving sets. Thus, $\operatorname{dim}(G)=2$ and $r_{\left(u_{1}, v_{1}\right)}(G)=$ $2, \quad r_{\left(u_{1}, v_{2}\right)}(G)=2, \quad r_{\left(u_{n}, v_{1}\right)}(G)=2, \quad r_{\left(u_{n}, v_{2}\right)}(G)=2$. Also, note that $W_{3}=$ $\left\{\left(u_{i}, v_{1}\right),\left(u_{i+1}, v_{1}\right),\left(u_{i}, v_{2}\right)\right\} \quad, \quad W_{4}=\left\{\left(u_{i}, v_{1}\right),\left(u_{i+1}, v_{1}\right),\left(u_{i+1}, v_{2}\right)\right\} \quad W_{5}=$ $\left\{\left(u_{i}, v_{2}\right),\left(u_{i+1}, v_{2}\right),\left(u_{i}, v_{1}\right)\right\}$ and $W_{6}=\left\{\left(u_{i}, v_{2}\right),\left(u_{i+1}, v_{2}\right),\left(u_{i+1}, v_{1}\right)\right\}$ are minimum resolving sets containing $\left(u_{i}, v_{1}\right),\left(u_{i+1}, v_{1}\right),\left(u_{i}, v_{2}\right),\left(u_{i+1}, v_{2}\right)$ respectively, for $2 \leq$ 
$i \leq(n-2)$.

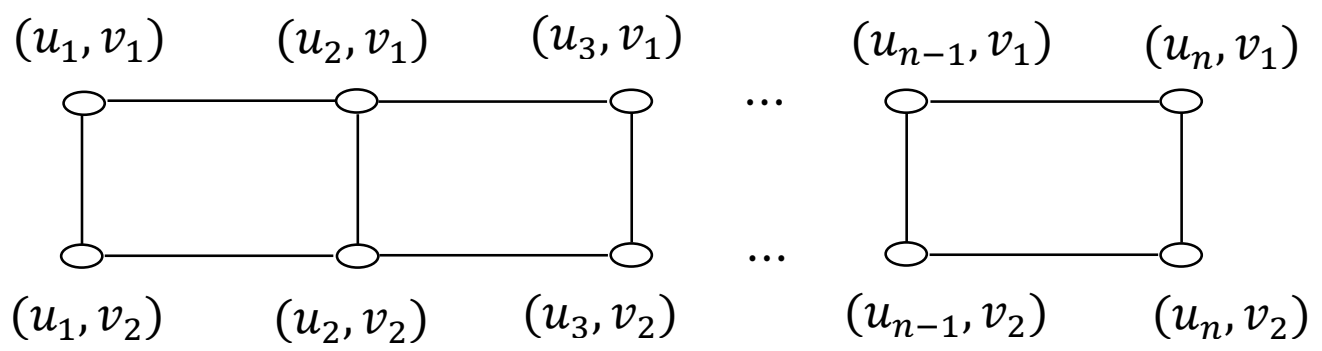

Fig. $4 \quad\left(P_{n} \times K_{2}\right)$

Thus, $r_{\left(u_{i}, v_{1}\right)}(G)=3$ and $r_{\left(u_{i}, v_{2}\right)}(G)=3$, for $2 \leq i \leq(n-1)$. Hence, $r_{a v}(G)=$ $3-\frac{2}{n}$ and hence $G=\left(P_{n} \times K_{2}\right)$ is not resolving excellent. Note that $\operatorname{dim}\left(P_{n} \times K_{2}\right)=$ $\operatorname{dim}\left(P_{n}\right)+1$ and $r_{a v}\left(P_{n} \times K_{2}\right)=r_{a v}\left(P_{n}\right)+1$

THEOREM 3.2. If $C_{n}$ is a cycle graph of length $n(n \geq 3)$ and $K_{2}$ is a path order 2, then $\left(C_{n} \times K_{2}\right)$ is resolving excellent.

Proof. Let $G=\left(C_{n} \times K_{2}\right),(n \geq 3)$ and $V(G)=\left\{\left(u_{1}, v_{1}\right),\left(u_{2}, v_{1}\right), \cdots,\left(u_{n}, v_{1}\right)\right.$, $\left(u_{1}, v_{2},\left(u_{2}, v_{2}\right), \cdots,\left(u_{n}, v_{2}\right)\right\}$.

Case 1: Let $n$ be odd. $W_{1}=\left\{\left(u_{i}, v_{1}\right),\left(u_{i+2}, v_{1}\right)\right\}$ and $W_{2}=\left\{\left(u_{i}, v_{2}\right),\left(u_{i+2}, v_{2}\right)\right\}$ are minimum resolving sets containing $\left(u_{i}, v_{1}\right)$ and $\left(u_{i}, v_{2}\right)$, for $1 \leq i \leq n$ respectively. Therefore, $\operatorname{dim}\left(C_{n}\right)=2, \operatorname{dim}\left(C_{n} \times K_{2}\right)=2, r_{(u, v)}\left(C_{n} \times K_{2}\right)=2$, for all $(u, v) \in V\left(C_{n} \times K_{2}\right)$ and hence $\left(C_{n} \times K_{2}\right)$ is resolving excellent. Note that $\operatorname{dim}\left(C_{n}\right)=$ $\operatorname{dim}\left(C_{n} \times K_{2}\right)=2$ and $r_{a v}\left(C_{n} \times K_{2}\right)=r_{a v}\left(C_{n}\right)$ for $n$ odd.

Case 2: Now, let us assume $n$ is even. $W_{1}=\left\{\left(u_{i}, v_{1}\right),\left(u_{i+1}, v_{1}\right),\left(u_{i}, v_{2}\right)\right\}, W_{2}=$ $\left\{\left(u_{i}, v_{1}\right),\left(u_{i+1}, v_{1}\right),\left(u_{i+1}, v_{2}\right)\right\}, W_{3}=\left\{\left(u_{i}, v_{2}\right),\left(u_{i+1}, v_{2}\right),\left(u_{i}, v_{1}\right)\right\}, W_{4}=\left\{\left(u_{i}, v_{2}\right)\right.$, $\left.\left(u_{i+1}, v_{2}\right),\left(u_{i+1}, v_{1}\right)\right\}$ are minimum resolving sets, for all $1 \leq i \leq(n-1)$. Thus, $\operatorname{dim}(G)=3$ and $r_{(u, v)}(G)=3$, for all $(u, v) \in V(G)$. Hence, $r_{a v}\left(C_{n} \times K_{2}\right)=3$ and $G=\left(C_{n} \times K_{2}\right)$ is resolving excellent. Note that $\operatorname{dim}\left(C_{n} \times K_{2}\right)=\operatorname{dim}\left(C_{n}\right)+1$, for $n$ even, and $r_{a v}\left(C_{n} \times K_{2}\right)=r_{a v}\left(C_{n}\right)+1$ for $n$ even.

Theorem 3.3. If $H$ is a connected graph and $K_{2}$ is a path of order 2, then $r_{u}(H) \leq$ $r_{(u, v)}\left(H \times K_{2}\right) \leq r_{u}(H)+1$, for $u \in H, v \in K_{2}$.

Proof. Let $G=H \times K_{2}$. Let $V(H)=\left\{u_{1}, u_{2}, \ldots, u_{n}\right\}, V\left(K_{2}\right)=\left\{v_{1}, v_{2}\right\}$ and $V(G)=$ $\left\{\left(u_{i}, v_{j}\right) \mid 1 \leq i \leq n, 1 \leq j \leq 2\right\}$. Let $S=\left\{u=u_{1}^{\prime}, u_{2}^{\prime}, \ldots, u_{k}^{\prime}\right\}$ be the minimum resolving set of $H$ containing $u$. Then we claim that $W=\left\{\left(u_{1}^{\prime}, v_{1}\right),\left(u_{2}^{\prime}, v_{1}\right)\right.$, $\left.\ldots,\left(u_{k}^{\prime}, v_{1}\right),\left(u_{1}^{\prime}, v_{2}\right)\right\}$ is the resolving set of $G$ containing $(u, v)$, for $v \in K_{2}$. First let us prove that $W$ is a resolving set. For, let $(w, x)$ and $(y, z)$ be two vertices of $G$ such that $r((w, x) \mid W)=r((y, z) \mid W)$. We show that the vertices $(w, x)$ and $(y, z)$ are one and the same. This is certainly true if $(w, x)$ or $(y, z)$ belongs to $W$. So, we assume that $(w, x),(y, z) \notin W$. That is, we consider the vertices of $V(G)-W$. We consider three cases.

Case 1: Both $x=z=v_{1}$. Then $d_{G}\left((w, x),\left(u_{i}^{\prime}, v_{1}\right)\right)=d_{H}\left(w, u_{i}^{\prime}\right)$ and $d_{G}\left((y, z),\left(u_{i}^{\prime}, v_{1}\right)\right)=d_{H}\left(y, u_{i}^{\prime}\right)$, for $i=1,2, \ldots, k$. Suppose $w \neq y$. Since $S$ is a 
resolving set for $\mathrm{H}$, it follows that $d_{H}\left(w, u_{i}^{\prime}\right) \neq d_{H}\left(y, u_{i}^{\prime}\right)$ for some $i \quad(1 \leq i \leq k)$. Hence $d_{G}\left((w, x),\left(u_{i}^{\prime}, v_{1}\right)\right) \neq d_{G}\left((y, z),\left(u_{i}^{\prime}, v_{1}\right)\right)$ which contradicts the fact that $r((w, x) \mid W)=r((y, z) \mid W)$. Therefore $w=y$ and hence $(w, x)$ and $(y, z)$ are one and the same.

Case 2: Either $x=v_{1}$ and $z=v_{2}$, or $x=v_{2}$ and $z=v_{1}$. Consider the former. In this case, $d_{G}\left((w, x),\left(u_{1}^{\prime}, v_{2}\right)\right)=d_{G}\left((w, x),\left(u_{1}^{\prime}, v_{1}\right)\right)+1$ and $d_{G}\left((y, z),\left(u_{1}^{\prime}, v_{2}\right)\right)=$ $d_{G}\left((y, z),\left(u_{1}^{\prime}, v_{1}\right)\right)-1$. Thus, either $d_{G}\left((w, x),\left(u_{1}^{\prime}, v_{2}\right)\right) \neq d_{G}\left((y, z),\left(u_{1}^{\prime}, v_{2}\right)\right)$ or $d_{G}\left((w, x),\left(u_{1}^{\prime}, v_{1}\right)\right) \neq d_{G}\left((y, z),\left(u_{1}^{\prime}, v_{1}\right)\right)$, contradicting the fact that $r((w, x) \mid W)=$ $r((y, z) \mid W)$. Thus, $(w, x)$ and $(y, z)$ are one and the same.

Case 3: Both $x=z=v_{2}$. First, suppose that atleast one of $w$ and $y$ belongs to $S$, say $w \in S$. Then $w=u_{i}^{\prime}$, for some $i(1 \leq i \leq k)$, so $d_{G}\left(\left(w, v_{2}\right),\left(u_{i}, v_{1}\right)\right)=1$. Since $r((w, x) \mid W)=r((y, z) \mid W)$, we have $d_{G}\left(\left(y, v_{2}\right),\left(u_{i}, v_{1}\right)\right)=1$. But, the only vertex in $\left\{\left(u_{i}, v_{2}\right) / 1 \leq i \leq n\right\}$ adjacent to $\left(u_{i}, v_{1}\right)$ is $\left(u_{i}, v_{2}\right)$; so, $\left(w, v_{2}\right)=\left(u_{i}, v_{2}\right)=\left(y, v_{2}\right)$.

If both $w, y \notin S$, consider the vertices $\left(w, v_{1}\right)$ and $\left(y, v_{1}\right)$. As in case 1 , if $\left(w, v_{1}\right) \neq$ $\left(y, v_{1}\right)$ then $d_{G}\left(\left(w, v_{1}\right),\left(u_{i}^{\prime}, v_{1}\right)\right) \neq d_{G}\left(\left(y, v_{1}\right),\left(u_{i}^{\prime}, v_{1}\right)\right)$ for some $i \quad(1 \leq i \leq k)$. Now, $d_{G}\left(\left(w, v_{2}\right),\left(u_{i}^{\prime}, v_{1}\right)\right)=d_{G}\left(\left(w, v_{1}\right),\left(u_{i}^{\prime}, v_{1}\right)\right)+1 \quad$ and $\quad d_{G}\left(\left(y, v_{1}\right),\left(u_{i}^{\prime}, v_{1}\right)\right)=$ $d_{G}\left(\left(y, v_{1}\right),\left(u_{i}^{\prime}, v_{1}\right)\right)+1$. Therefore, $d_{G}\left(\left(w, v_{1}\right),\left(u_{i}^{\prime}, v_{1}\right)\right)=d_{G}\left(\left(y, v_{1}\right),\left(u_{i}^{\prime}, v_{1}\right)\right)$, which implies $w=y$.

Hence, $W$ is the resolving set and $r_{(u, v)}\left(H \times K_{2}\right) \leq r_{u}(H)+1$, for $u \in H, v \in K_{2}$.

Now, we prove the lower bound stated in the theorem. Let $\left(u, v_{1}\right) \in H \times K_{2}$ and $W$ be the minimum resolving set of $G$ containing $\left(u, v_{1}\right)$. Let $W_{1}=\left\{x \in H \mid\left(x, v_{1}\right) \in W\right\}$ and $W_{2}=\left\{x \in H \mid\left(x, v_{2}\right) \in W\right\}$. Let $S \subseteq V(H)$ be the union of $W_{1}$ and $W_{2}$. Then, $|S|=\left|W_{1} \cup W_{2}\right| \leq\left|W_{1}\right|+\left|W_{2}\right|=|W|$ Clearly $u \in S$ and we claim that $S$ is the minimum resolving set for $H$ containing $u$. Let $x$ and $y$ be distinct vertices of $H$. We show that $r(x \mid S) \neq r(y \mid S)$. If either $x$ or $y$ belongs to $W$, then this is certainly the case. Otherwise, there exists a vertex $(w, v) \in W$ such that $d_{G}((x, v),(w, v)) \neq$ $d_{G}((y, v),(w, v))$. If $\quad v=v_{1} \quad$, then $d_{H}(x, w)=d_{G}\left(\left(x, v_{1}\right),\left(w, v_{1}\right)\right) \neq$ $d_{G}\left(\left(y, v_{1}\right),\left(w, v_{1}\right)\right)=d_{H}(y, w)$. If $v=v_{2}$, then $d_{H}(x, w)=d_{G}\left(\left(x, v_{1}\right),\left(w, v_{2}\right)\right)-$ $1 \neq d_{G}\left(\left(y, v_{1}\right),\left(w, v_{2}\right)\right)-1=d_{H}(y, w)$.In either case, $r(x \mid S) \neq r(y \mid S)$ and hence $r_{u}(H) \leq r_{(u, v)}\left(G \times K_{2}\right)$.

COROLLARY 3.3.1 If $H$ is a connected graph and $K_{2}$ is a path of order 2, then $r_{a v}(H) \leq r_{a v}\left(H \times K_{2}\right) \leq r_{a v}(H)+1$.

\section{AVERAGE RESOLVING NUMBER OF BISMUTH TRI-IODIDE CHAIN ( $\left.m-B_{i} I_{3}\right)$ AND AVERAGE RESOLVING NUMBER OF $m$-Lead Chloride Chain $\left(m-\mathrm{PbCl}_{2}\right)$}

Crystal structure is one of the most important aspects of materials science and engineering as many properties of materials depend on their crystal structures. Understanding the basics of crystal structures is of paramount importance in crystallography.

A crystal or crystalline solid is a solid material whose constituents (such as atoms,ions, or molecules) are arranged periodically in a definite repetitive patten. The unit cell is the small repeating entity of the atomic structure.

Bismuth Tri-Iodide $\left(\mathrm{BiI}_{3}\right)$ is an inorganic compound. This grey-black solid is the result of the reaction of bismuth and iodine and it was once the interest of qualitative inorganic analysis [12]. A solitary precious stone of $\mathrm{BiI}_{3}$ has been integrated by Nason and Keller [13]. The graph of one unit of bismuth tri-iodide is shown in Fig.5(a). It contains six 4-cycles of which two are on the top, two are in the centre and two at the base. If $m$ unit 
cells of bismuth tri-iodide are arranged linearly, then it is called an $m$-bismuth chain.A 2-Bismuth Chain is shown in Fig.5(b). Various parameters of $m$-bismuth chain exists in the literature. For instance. metric dimension and fault-tolerant metric dimension of $m$-bismuth chain[14], closed forms of redefined first, second and third Zagreb indices [15], degree-based added-substance topological indices principally the first, second, and hyper Zagreb indices [16] to name a few.

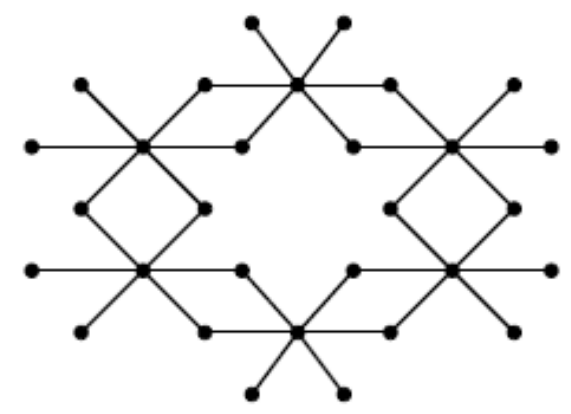

Fig. 5 (a) One Unit of Bismuth Tri-Iodide

The number of vertices, the number of 1-degree vertices, 2-degree vertices and 6-degree vertices in $m$ - bismuth chain are $6(3 m+2), 4(m+2), 2(5 m+1)$ and $2(2 m+1)$ respectively. The cycles in an $m$-bismuth chain are labelled as $C_{i}^{T}$ (Top Cycles), $C_{j}^{C}$ (central Cycles) and $C_{i}^{B}$ (Base Cycles), $1 \leq i \leq 2 m$ and $1 \leq j \leq m+1$. Let $p_{k}, p_{k}^{\prime}, q_{k}, q_{k}^{\prime}, 1 \leq i \leq m+2, x_{i}, x_{i}^{\prime} \in C_{i}^{T}, y_{j}, y_{j}^{\prime} \in C_{j}^{C}$ and $z_{i}, z_{i}^{\prime} \in C_{i}^{B}$. We now find the average resolving number of $m$-bismuth chain.

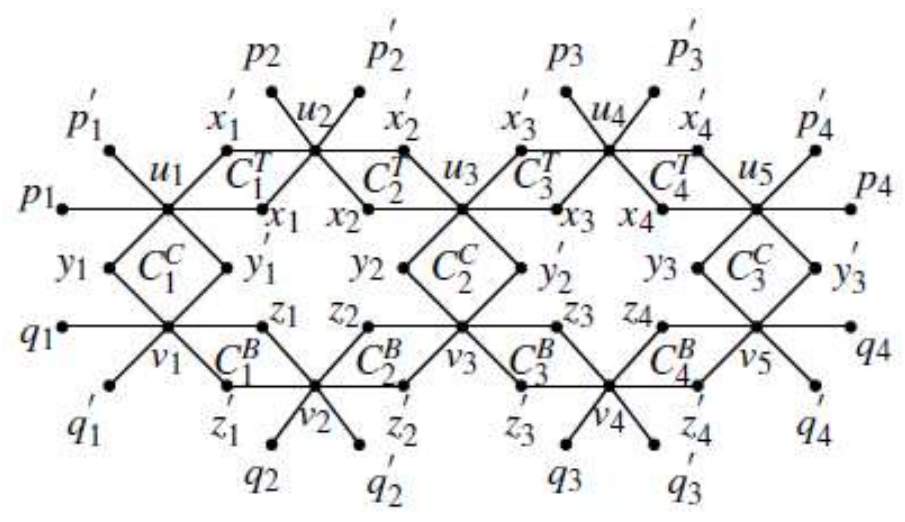

Fig. 5 (b) A 2-Bismuth Chain

THEOREM 4.1. Let $G$ be an $m$-bismuth chain. Then $r_{a v}(G)=\operatorname{dim}(G)+\frac{2 m+1}{9 m+6}$.

Proof. The metric dimension of $G$ is $7 m+5$ and the set $W$, consists of a 2-degree vertex from each 4-cycle $\left(C_{i}^{T}, C_{j}^{C}, C_{i}^{B}\right)$ and a leaf vertex from each of the pair of leaf incident with a 4-cycle(either $p_{k}$ or $p_{k}^{\prime}$ and either $q_{k}$ or $q_{k}^{\prime}$ ) is a minimum resolving set of $G[14]$. Moreover, every resolving set of $m$-bismuth chain should contain this minimum resolving set $W$ as the vertices in $W$ are equidistant from every vertex of $G$. Thus, $r_{u}(G)=7 m+5$ for all $u \in W$. Let $W^{*}=W \cup\{v\}$ for $v \in V \backslash W$. Since $W^{*}$ is a super set of $W$ and every resolving set of $G$ should contain $W, W^{*}$ is minimum resolving set containing $v \in V \backslash W$. Thus, $r_{v}(G)=7 m+6$ for all $v \in V \backslash W$ and hence $r_{a v}(G)=\frac{(7 m+5)(14 m+10)+(7 m+6)(4 m+2)}{18 m+12}=\operatorname{dim}(G)+\frac{2 m+1}{9 m+6}$. Note that the $m$-bismuth 
chain is not resolving excellent for any $m$.

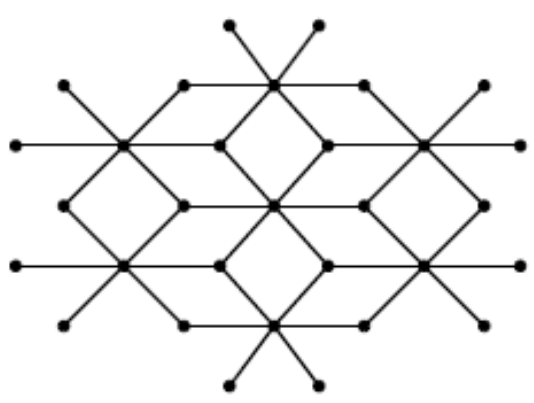

Fig. 6 (a) One unit of Lead Chloride

Lead Chloride $\left(\mathrm{PbCl}_{2}\right)$ is a halide crystal and is one of the most important lead-based reagents. It also occurs naturally in the form of mineral cotunnite and its structure is orthorhombic dipyramidal. The graph of a single unit of lead chloride is obtained from that of the single unit of bismuth tri-iodide by joining just one 2-degree vertex of each of the 4-cycles to a new vertex as shown in Fig. 6(a). As in the case of bismuth tri-iodide, the linear arrangement of $m$ unit cells is called an $m$-lead chloride chain.

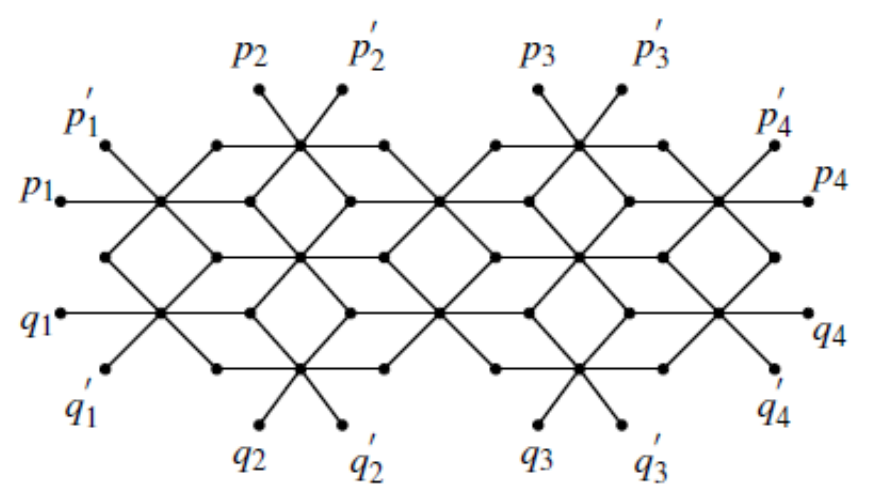

Fig. 6 (b) A 2-Lead Chloride Chain

A 2-lead chloride chain is given in Fig.6(b). The number of vertices in $m$-lead chloride chain is $19 m+12$ and the number of 1 -degree vertices in $m$-lead chloride chain is same as that of the number of 1-degree vertices in $m$-bismuth chain. Now, we find the average resolving number of $m$-lead chloride chain.

THEOREM 4.2. Let $G$ be an $m$-lead chloride chain. Then $r_{a v}(G)=\operatorname{dim}(G)+$ $\frac{23 m+4}{19 m+12}$.

Proof.Let $p_{i}, q_{i}, p_{i}^{\prime}, q_{i}^{\prime}, 1 \leq i \leq m+2$ be the 1-degree vertices as shown in Fig.6(b). The metric dimension of $G$ is $2 m+4$ and the set $W$ consists of a leaf vertex from every pair 1-degree vertices (i.e., either $p_{k}$ or $p_{k}^{\prime}$ and either $q_{k}$ or $q_{k}^{\prime}$ ) incident with a common vertex is a minimum resolving set and every resolving set of $G$ should contain this minimum resolving set $W$ as the vertices in $W$ are equidistant from every vertex of $G$ [14]. Thus, $\operatorname{dim}(G)=2 m+4$ and $r_{u}(G)=2 m+4$, for all $u \in W$. Let $W^{*}=$ $W \cup\{v\}$ for $v \in V \backslash W$. Since $W^{*}$ is a super set of $W$ and every resolving set of $G$ should contain $W, W^{*}$ is minimum resolving set containing $v \in V \backslash W$. Thus, $r_{v}(G)=$ $2 m+5$ for all $v \in V \backslash W$ and hence $r_{a v}(G)=\frac{(2 m+4)(4 m+8)+(2 m+5)(15 m+4)}{19 m+12}=$ 
$\operatorname{dim}(G)+\frac{23 m+4}{19 m+12}$. Clearly, the $m$-lead chloride chain is not resolving excellent for any $m$.

\section{CONCLUSION}

In this paper, we have defined a new resolving parameter, called resolving number, $r_{a v}(G)$ and found the average resolving number of some standard graphs, product graphs, $m$-bismuth chain and $m$-lead chloride chain.The use of this new parameter have been explained using the chemical graph of Butane and its structural isomer called isobutane.We found the bounds for $r_{a v}(G)$ in terms of $\operatorname{order}(n)$ and diameter( $(d)$ of the graph $G$. We have also defined the concept of resolving excellent graphs and examined the above mentioned graphs are resolving excellent or not.For future work, we provide the following problems as follows.

PROBLEM 5.1 For $n>2$, let $H=G \times P_{n}$, where $G$ is a connected graph. Determine the relation between $r_{a v}(G)$ and $r_{a v}(H)$.

PROBLEM 5.2 For $n \geq 3$, let $H=G \times C_{n}$, where $G$ is a connected graph. Determine the relation between $r_{a v}(G)$ and $r_{a v}(H)$.

PROBLEM 5.3 Characterize all resolving excellent graphs.

\section{REFERENCES}

[1] P. J. Slater, "Leaves of trees". Congr. Numer., 14:549-559 (1975).

[2] F. Harary and R. A. Melter, "On the metric dimension of a graph". Ars Combin., 2:191-195 (1976).

[3] G. Chartrand, C. Poisson, P. Zhang, "Resolvability and the upper dimension of graphs", Comput. Math. Appl. 39: 19-28(2000)

[4] Chartrand, G., L. Eroh, M.A. Johnson and O.R. Oellermann, , "Resolvability in graphs and the metric dimension of a graph", Disc. Appl. Math.. 105:99-113(2000)

[5] Chartrand, G. and Zhang, P. "The theory and application of resolvability in graphs: A survey", Congr.Numer., Vol.160, pp.47-68(2003)

[6] Sebo,A. and Tannier,E. "On metric generators of graphs", Math. Oper. Res., Vol.29, pp.383-393(2004)

[7] Z. Beerliova, F. Eberhard, T. Erlebach, A. Hall, M. Hoffmann, M. Mihal'ak, and L. Ram. "Network discovery and verification". IEEE J. on Selected Areas in Comunications, 24(12):2168-2181 (2006).

[8] Khuller, S., Raghavachari, B and Rosenfield, A. "Landmarks in graphs", Discrete Appl. Math., Vol.70, pp.217-229 (1996)

[9] Chavatal, V. "Mastermind", Combinatorica, Vol.3, pp.325-329 (1983)

[10] U. Ali, S. A. Bokhary, K. Wahid and G. Abbas, "On resolvability of a graph associated to a finite vector space". Journal of Algebra and Its Applications, Vol 18, No.02(2019).

[11] Hafiz Muhammad Afzal Siddiqui and Muhammad Imran, "Computing Metric and Partition Dimension of 2-Dimensional Lattices of Certain Nanotubes". Journal of Computational and Theoretical Nanoscience. 11. 10.1166/jctn.2014.3656 (2014)

[12] Imran, M., Ali, M. A., Ahmad, S., Siddiqui, M. K. and Baig, A. Q. "Topological Characterization of the Symmetrical Structure of Bismuth Tri-Iodide". Symmetry 10, 201; doi:10.3390/sym10060201 (2018)

[13] Nason, D.; Keller, L. "The Growth and Crystallography of Bismuth Tri-Iodide Crystals Grown by Vapor Transport". J. Cryst. Growth 221 (1995)

[14] Krishnan, Sathish \& Rajan, Bharati. "Fault-Tolerant Resolvability of Certain Crystal Structures". Applied Mathematics. 07. 599-604. 10.4236/am.2016.77055. (2016)

[15] Abaid ur Rehman Virk, Waqas Nazeer and Shin Min Kang "On "computational Aspects of Bismuth Tri-Iodide" 10.20944/preprints201806.0209.v1.

[16] Muhammad Imran, Muhammad Arfan Ali, Sarfraz Ahmad, Muhammad Kamran Siddiqui and Abdul Qudair Baig," Topological Characterization of the Symmetrical Structure of Bismuth Tri-Iodide" Symmetry 2018, 10(6), 201 